\title{
Visibilidad e impacto científico: el caso de las revistas colombianas de economía
}

\author{
Joan-Miguel Tejedor-Estupiñán*
}

Ricardo-Alonso Tejedor-Estupiñán**

Artículo recibido:

14 de febrero de 2018

Artículo aceptado:

29 de octubre de 2018

Artículo de investigación

\section{Resumen}

Se identifican las revistas colombianas de ciencias económicas mediante un análisis comparativo de diferentes indicadores bibliométricos como el IBN de Publindex, el índice de Hirsch (índice- $h$ ), el CiteScore de Scopus y el Scimago Journal Report de Elsevier. Los resultados evidencian su adaptación al proceso de globalización del conocimiento y la información, por medio de su visibilidad e impacto tanto nacional como internacional, en un contexto de relaciones asimétricas en el flujo y acceso a la información entre países desarrollados y en vías de desarrollo. Las revistas identificadas sirven de referencia para investigadores, autores,

* Universidad Católica de Colombia

jmtejedor@ucatolica.edu.co

** Universidad Pedagógica y Tecnológica de Colombia ricardotse@hotmail.com 
editores, profesionales del campo de las ciencias económicas, diseñadores de políticas y demás interesados en la gestión de la información.

Palabras Clave: Revistas Colombianas; Ciencias Económicas; Indicadores Bibliométricos; Bases de Datos; Investigación y Desarrollo

Visibility and Scientific Impact: the case of Colombian Journals of Economics

Joan-Miguel Tejedor-Estupiñán and Ricardo-Alonso Tejedor-Estupiñán

\section{Abstract}

Colombian journals of economic sciences are identified through a comparative analysis of different bibliometric indicators, such as the Publindex National Bibliographic Index, Hirsch's index (h-index), the Scopus's Cite Score and Scimago Journal Report from Elsevier's. The results show their adaptation to the process of globalization of knowledge and information, through their visibility and impact at national and international levels, in a context of asymmetric relations in the flow and access to information between developed and developing countries. The journals identified, serve as a high-quality source for researchers, authors, editors and professionals in the field of economics, policymakers and others interested in information management.

Keywords: Colombian Journals; Economics; Bibliometric Indicators; Databases; Information; Research and Development

\section{INTRODUCCIÓN}

$\mathrm{Z}_{\mathrm{y} \text { el conocimiento, caracterizado por las relaciones asimétricas interde- }}^{\mathrm{n} \text { un escenario de globalización, desde la dimensín de la informacion }}$ pendientes entre países desarrollados y en vías de desarrollo, en especial las relacionadas al flujo y acceso de la información (Szentes, 2003), se destaca la gestión editorial realizada por las instituciones, equipos editoriales y redes científicas que editan las revistas de ciencias económicas en Colombia. 
Al identificar las revistas colombianas de ciencias económicas en diferentes bases de datos, y comparando distintos indicadores para medir el impacto de una revista como el IBN de Publindex, el índice de Hirsch (índice- $b$ ), el CiteScore de Scopus y el Scimago Journal Report (SJR) de Scimago, el objetivo de este documento es conocer cuál es la visibilidad y el nivel de impacto internacional de las revistas colombianas de ciencias económicas en el periodo 1999-2017; igualmente, discutir los retos que enfrentan actualmente las revistas colombianas.

Los métodos bibliométricos permiten evaluar el impacto de las revistas científicas por medio de variables como producción, citación, productividad, influencia científica, disciplina, cooperación interinstitucional y/o internacional, e identifican tendencias en las investigaciones, conformación de redes, clasificación de autores, instituciones, revistas, países, etc. (Sánchez Perdomo et al., 2018). Los estudios bibliométricos contribuyen al diseño de políticas de investigación y desarrollo, en particular las relacionadas con la divulgación científica de entidades públicas y privadas, así como las políticas laborales que afectan a investigadores y docentes (Ordóñez M. et al., 2009; Álvarez-Muñoz y Pérez-Montoro, 2015).

Los creadores de la Red de Revistas Científicas de América Latina y el Caribe (Redalyc) proponen una alternativa iberoamericana, preocupada por la divulgación científica y la generación de datos para elaborar análisis bibliométricos, con revistas de estas regiones que no se incluyen en el Journal Citation Report (JCR) o en SJR. Esta alternativa critica la baja participación y posibilidad de acceso de las revistas latinoamericanas y periféricas en estas bases de datos, cuestionando el modelo oligopólico que éstas promueven (Aguado López y Rogel Salazar, 2006; Aguado-López et al., 2008).

Sobre Latinoamérica, encontramos el estudio de Sánchez Perdomo et al. (2018) acerca del impacto de la producción científica relacionada con ciencias de la información por medio del análisis de la base de datos de Scopus. Babini (2011) compara varios indicadores regionales e internacionales e identifica las principales instituciones de la región de acuerdo a su producción científica y visibilidad en línea, destacando principalmente el acceso abierto a sus contenidos. Miguel (2011) desarrolla una comparación, por país y por tema, de la cobertura de revistas de América Latina y el Caribe, incluidas en Scientific Electronic Library Online (SciELO), la hemeroteca de acceso libre Redalyc y Scopus, estimando el volumen de la producción científica y su evolución en el periodo 2005-2009. Igualmente, Montilla-Peña (2012) analiza la producción científica archivística registrada en Redalyc.

Álvarez-Muñoz y Pérez-Montoro (2015) realizan un análisis cuantitativo de la producción científica ecuatoriana durante el periodo 2000-2013; 
basados en el SJR, evidencian el creciente desempeño de este país latinoamericano en términos de producción científica y colaboración internacional. Abad-García et al. (2015) presentan un estudio sobre las características y visibilidad de 207 revistas españolas de ciencias de la salud activas en 2014 y su visibilidad en bases de datos, destacando el incremento de las publicaciones bilingües, online y de acceso abierto.

El estudio de Harzing y Van der Wal (2009) presenta un análisis del impacto de las revistas de ciencias económicas y negocios, comparando el índice- $b$ de Google como alternativa al Journal Impact Factor (JIF), afirmando que el primero es una excelente alternativa que cuenta con mayor cobertura e incluye documentos no citables, y que la citación por otros académicos no es la única medida relevante para calcular el impacto de la investigación y las publicaciones. El estudio de Espinosa, Restrepo y Rodríguez (2003), mediante el método de clasificación temática de Alan Williams, evidencia la baja producción en el área de economía de la salud. Igualmente, Ordóñez et al. (2009) identifican las fortalezas y debilidades de la Revista de Economía Institucional (REI) y su desempeño e impacto en sus primeros 10 años de existencia.

Finalmente, Romero-Torres, Acosta-Moreno y Tejada-Gómez (2013) presentan una propuesta para homologar el sistema nacional de Publindex al sistema internacional, dado que gran parte de las revistas colombianas clasificadas en las categorías A1, A2, B y C de la Base Bibliográfica Nacional Publindex (BBN) no forman parte de las bases bibliográficas internacionales de Elsevier (Scopus) y de Web of Science (WoS, de Clarivate Analytics). La investigación de Marenco-Escuderos (2013) analiza variables como los tipos de colaboración, las áreas de conocimiento y los tipos de artículo publicados para una revista de psicología en Colombia.

\section{MATERIALES Y MÉTODOS}

Encontramos el factor de impacto (FI o JIF), presentado en 1960 por Irving H. Sher y Eugene Garfield para el Instituto para la Información Científica (ISI) de Filadelfia (cuyos productos más importantes son Web of Science, Journal Citations Reports y Web of Knowledge, los cuales fueron comprados por Clarivate Analytics en 2016). Este indicador evalúa las revistas científicas, dividiendo el número de citaciones de los artículos publicados en una revista durante los dos últimos años por el número de artículos (citables) publicados durante el mismo tiempo. Sólo se incluyen como citables (denominador) las referencias citadas de artículos originales pero no los artículos de revisión, editoriales o cartas al editor, publicados en las revistas indexadas (incluidas en el SCI) (Garfield, 1994, 2006; Beltrán-Galvis, 2006). 


\section{FI= Citas Recibidas de Artículos Recientes \\ Número de Artículos Publicados}

Aunque al principio el FI fue destacado como técnica para la evaluación científica, ha sido criticado por las limitaciones para evaluar la calidad de un artículo y el impacto real de la ciencia. Por ejemplo: de acuerdo al área, dado que las revistas de ciencias básicas tienen mayor FI que las de ciencias sociales; según el tipo de artículo, los documentos de revisión tienen más FI que los documentos de innovación e investigación; según el idioma, ya que las revistas en inglés tienen mayor FI, así como según la ubicación geográfica, ya que las revistas de USA tienen mayor FI que las de Europa (Garfield, 1994, 2006; Beltrán-Galvis, 2006). Otras críticas en contra del FI argumentan que no mide los procesos de calidad en la edición de la revista, como la evaluación; no mide la tasa de citación de artículos individualmente y puede ser manipulado por los editores, por lo tanto es necesario abandonar su uso y plantear nuevos indicadores, e incluso renombrarlo como "índice de tasa de citaciones", debiéndose evitar su uso como indicador para medir el verdadero impacto de una revista, o usarse como referencia para validar investigadores, centros de investigación, presupuestos, políticas, etc. (Hecht, Hecht y Sandberg, 1998; Archambault y Larivière, 2009; Kurmis, 2003). En este documento no se analiza este indicador por las anteriores razones y dada la incipiente participación de revistas colombianas de ciencias económicas en este ranking.

El SJR es un ranking que establece la calidad de las publicaciones científicas basándose en el recuento de citas obtenidas por cada revista, usando como fuente la base de datos de Scopus. El SJR resulta del cálculo de las citas recibidas por las revistas en un periodo de tres años, y otorga un peso mayor a las citas procedentes de revistas de alto prestigio (las cuales posean baja autocitación y altas tasas de citación), apoyándose en el uso del algoritmo de Google PageRank (Guerrero-Bote y Moya-Anegón, 2012). El SJR incluye la clasificación por cuartiles, el cual es un indicador que muestra la medida de posición de una revista en relación a otras de su área. En el primer cuartil se ubican las revistas de mayor factor de impacto, el segundo y el tercero son los cuartiles medios y el más bajo el cuartil cuarto.

El indicador SJR es un recurso de acceso abierto, mientras que el IF requiere del pago por suscripción; asimismo, el SJR contiene más revistas que el IF, cubriendo igualmente más regiones y lenguajes. Los dos índices dividen las citaciones de una revista por los artículos de la misma durante un periodo de 2 a 5 años. Sin embargo, a diferencia del IF, el SJR otorga más importancia a las revistas dependiendo de su prestigio. Mientras el SJR calcula todos los documentos en el denominador, el IF sólo calcula los documentos "citables" (Falagas et al., 2008). 
Hirsch propone un método alternativo para medir el impacto de la producción científica acumulada por los investigadores, llamado índice de Hirsch o índice- $b$, el cual representa el número aplicado a un investigador que tiene $b$ trabajos que han sido citados al menos $b$ veces en un periodo de tiempo específico. En otras palabras, consiste en consolidar los trabajos de un autor y ordenarlos en forma descendente en función de las citas recibidas. Como cada trabajo tiene una cantidad de citas, también posee un número de orden en el ranking, denominado rango; como resultado tenemos dos listas de números, una ascendente (los rangos) y una descendente (las citas). La intersección entre los valores de ambas es lo que se denomina índice- $h$. Dado que su proyección no es lineal, avanzar en la escala de valores es difícil, por lo que mejorar el umbral depende del aumento en la distribución de citas (Grupo Scimago, 2006). El índice $b$ abarca: a) número de trabajos, b) número total de citas, c) número de citas medias por trabajo, d) número de trabajos “significativos”, y e) número de citas de trabajos más citados (Hirsch, 2005).

Los académicos Harzing y Van der Wal (2009) han promovido el Google Scholar y el índice- $b$ como alternativas para la evaluación de las revistas de economía, asegurando que el primero proporciona una medida más precisa y completa del impacto de una revista debido a las limitaciones con respecto a subdisciplinas que tienen índices como el FI. Por su parte, Palucci-Marziale (2011) discute la pertinencia de usar indicadores para evaluar la calidad de la producción científica, como el JCR de Clarivate Analytics, el SJR y el Ranking Ibero Americano (SIR) desarrollado por Scopus, debido a sus limitantes como indicadores bibliométricos. Finalmente, Tol (2009) propone dos alternativas al índice- $h$, las cuales denomina índice- $f$ e índice- $t$, y Bartneck y Kokkelmans (2011) y Salvador-Oliván, Marco-Cuenca y Arquero-Avilés (2018) comprueban cómo los editores y los autores pueden manipular el índice- $h$ acudiendo a las autocitas y proponen el índice- $q$ como estrategia para detectar posible manipulación del índice- $h$, sobre los cuales no profundizaremos aquí.

Los datos sobre la clasificación nacional se obtuvieron del Índice Bibliográfico Nacional de Publindex (IBN), conformado por las Revistas Científicas Colombianas, clasificadas en las categorías A1, A2, B o C según el cuartil en que se encuentren en el ranking de SJR y el índice- $b$ de Google (Charum, 2004; Colciencias, 2013). Los datos del SJR se tomaron del portal de Scimago Journal and Country Rank (SJCR), quienes usan como fuente los datos de Scopus. El CiteScore se consultó directamente de la página de Scopus desarrollada por Elsevier. El índice- $h_{5}$ se consultó en Google Scholar. Finalmente, la participación en otras hemerotecas y directorios bibliométricos se verificó directamente en portales y bases de datos de EconLit (Journal List), Ideas de RePEc, Redalyc, SciELO, Ebsco y Proquest. Para encontrar las revistas indizadas en WoS se recurrió al Social Sciences Citation Index, publicado por Clarivate Analytics. 


\section{Resultados}

Como se aprecia en la Figura 1, la consolidación de la primera política de Publindex para 2015 había generado una expansión de revistas científicas en Colombia, pero al homologar el índice de acuerdo a estándares internacionales, la última convocatoria evidenció que muchas de estas publicaciones no tienen impactos significativos a nivel internacional (Colciencias, 2016; Rodríguez, Naranjo y González, 2015).

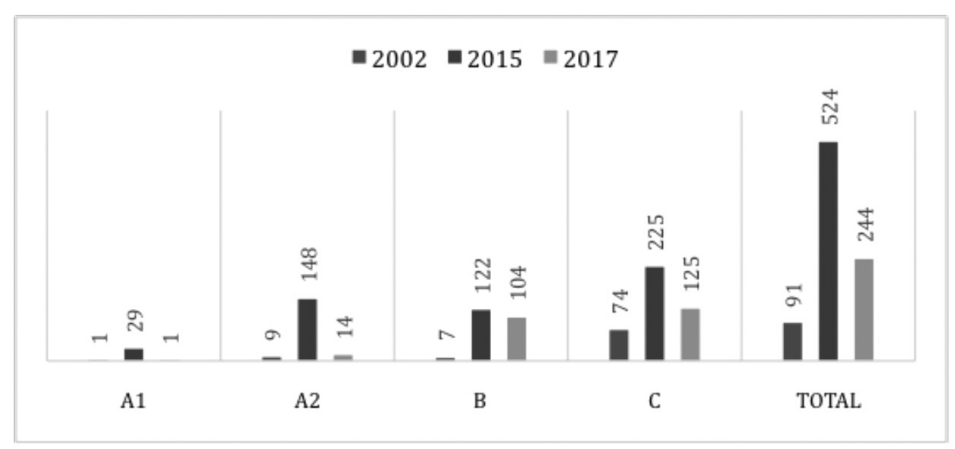

Figura 1. Revistas colombianas clasificadas por Publindex 2002, 2015, 2017

Con relación a las revistas de ciencias económicas, en 2014 Publindex reconoció 67 revistas $(12.7 \%$ ), dentro de las cuales dos se ubicaron en categoría A1, 22 en A2, 10 en B y 33 en C (Colciencias, 2016). De estas revistas 10 son anuales, 51 semestrales, tres trimestrales y tres cuatrimestrales. En 2017, el nuevo IBN de Publindex reconocía 20 revistas de ciencias económicas $(8.1 \%), 12$ fueron clasificadas en B y ocho en C (Anexo 1) (Colciencias, 2017 y y 2017b). Esto significa que estas revistas tienen un índice- $b$ que se encuentra en los cuartiles 3 y 4 de los cálculos de Publindex o se encuentran en Q3 y Q4 en SJR. En la primera actualización de Publindex 2014, alrededor de 26 revistas de ciencias económicas perdieron su clasificación en Publindex, en la segunda actualización de 2016, 47 revistas de ciencias económicas colombianas perdieron su clasificación.

Desde 1999, en el ranking SJR predominan las publicaciones de Europa (primordialmente Europa occidental) y Norteamérica, evidenciando las relaciones asimétricas entre países, relacionadas con el flujo y acceso a la información (Anexo 2). Se destaca la expansión desproporcionada y la concentración de las publicaciones en USA, el incremento en las regiones asiática y Europa del este, así como la escasa participación de las publicaciones de 
las regiones pacífica, africana y latinoamericana; esta última en 2017 apenas alcanzó un $2.5 \%$ del total de revistas reconocidas por el SJR (SCImago Journal \& Country Rank, 2018).

Dichas asimetrías entre países también se evidencian en la poca participación de revistas colombianas de ciencias económicas en las bases de datos de WoS, EconLit y RePEc (Tabla 1). Sin embargo, es importante resaltar que Colombia, junto con Brasil, son líderes en la región. De acuerdo a las revistas clasificadas en el SJR, los países latinoamericanos con más revistas de ciencias económicas en SJR son Brasil (ocho), Colombia (siete), México (cuatro) y Chile (cinco), la revista colombiana mejor posicionada en este índice es Cuadernos de Desarrollo Rural de la Universidad Javeriana (Anexo 3 ).

\begin{tabular}{|l|c|c|}
\hline \multicolumn{1}{|c|}{ Base de datos } & $\begin{array}{c}\text { Cobertura ciencias } \\
\text { económicas }\end{array}$ & $\begin{array}{c}\text { Revistas colombianas } \\
\text { de CE }\end{array}$ \\
\hline Scopus & 1006 & 8 \\
\hline WoS-SSCl & 799 & 1 \\
\hline EconLit & 750 & 17 \\
\hline RePEC & 2992 & 20 \\
\hline
\end{tabular}

Tabla 1. Cobertura de las revistas colombianas de economía en bases de datos (2017)

En 2017, el SCImago Journal Citation Rank Report clasificaba 34171 revistas de todo el mundo, entre estas, 842 latinoamericanas $(2.5 \%)$ y 99 colombianas $(0.28 \%)$. En el área general de economía, econometría y finanzas del SJR encontramos 1006 revistas, 29 (2.9\%) latinoamericanas y ocho (0.8\%) colombianas. Las revistas colombianas de economía que encontramos en este ranking se ubican en Q3 y Q4, es decir, en los grupos de revistas de impacto medio y bajo (SCImago Journal \& Country Rank, 2018). En el Anexo 4 se clasifican las revistas colombianas de ciencias económicas indexadas en Scopus (2018); al compararlas con otros indicadores, éstas tienen un mejor índice de citaciones en Google Scholar debido a que el índice- $b$ calculado es de 5 años y que además cuenta con su propia base de datos de revistas científicas.

El JCR de 2017 reconoce 11459 revistas, 15 colombianas de las cuales dos son de economía: Academia: Revista Latinoamericana de Administración (Social Science Citation Index-SSCI) del Consejo Latinoamericano de Escuelas de Administración - CLADEA, y Revista Cuadernos de Desarrollo Rural (Social Citations Index Expanded-SCIE) de la Universidad Javeriana de Colombia (Web of Science, 2017). Por otro lado, con la creación del Emerging Source Citation Index (ESCI) de WoS, ha surgido un índice que recoge revistas regionales que se integran de esta manera al proceso de evaluación para ascender a la colección central. En el último año ESCI ha indexado varias 
revistas colombianas de ciencias económicas como Finanzas y Política Económica (incluida en Scopus en octubre de 2017), Cuadernos de Economía, Clio América y Apuntes del CENES.

La visibilidad internacional de las revistas de ciencias económicas colombianas en otras colecciones diferentes a Scopus o WoS es evidente en otras bases de datos bibliográficas internacionales. El Anexo 5 ilustra el índice- $b_{5}$ para las revistas no indexadas en Scopus, pero reconocidas en el IBN de Publindex, destacando su participación en otras bases de datos. Aunque no se encuentra en Scopus, Estudios Gerenciales se muestra como la revista con mayor índice de citación, mientras que Apuntes del CENES, Económicas CUC, Clio América y Equidad y Desarrollo son las de menor impacto para Colombia (Ensayos de Economía y Panorama Económico no aparecen en Google Scholar).

De las 20 revistas de economía que se encuentran en B y C de Publindex, 17 se encuentran disponibles en el Listado de Revistas de EconLit, perteneciente a la American Economic Asociation. La colección de RePEc actualmente cubre 2992 revistas y sólo seis de las revistas colombianas de ciencias económicas reconocidas por Publindex no hacen parte de esta base de datos. Redalyc cuenta con una colección de 1258 revistas (de estas 212 son colombianas), en el área de economía existen 45 revistas, entre estas 11 son colombianas. La colección de SciELO cuenta con 1285 revistas activas, de las cuales 278 son colombianas, y 16 pertenecen al área de economía. Finalmente, sólo tres revistas no tienen sus contenidos en Ebsco y ocho no están disponibles en Proquest.

Desde un plano internacional, los datos de la evolución del gasto en los sectores de investigación y desarrollo $(\mathrm{I}+\mathrm{D})$ en tres países de la región andina, Colombia, Ecuador y Perú, muestran a Ecuador como el caso más destacable, aumentado su gasto en este sector en casi 0.4 puntos porcentuales. Mientras Perú se mantiene sobre el $1.2 \%$ de inversión en $\mathrm{I}+\mathrm{D}$, Colombia apenas pasó de $0.12 \%$ a $0.24 \%$ en 15 años (Banco Mundial, 2018). Con relación al gasto público en educación, Colombia apenas ha aumentado un punto porcentual entre 2000 y 2015, y mientras que Perú no alcanza un aumento de un punto, Ecuador incrementó significativamente el gasto público en educación, pasando de $1.5 \%$ a $4.9 \%$ en el mismo periodo. Esto muestra el liderazgo en términos de inversión en educación e I+D de Ecuador desde poco más de una década.

A pesar de que en Colombia no se desarrollan inversiones significativas en los sectores de $\mathrm{I}+\mathrm{D}$ y educación, la participación de sus revistas en los índices y bases bibliográficas internacionales es mucho mayor que Perú y Ecuador, quienes han aumentado significativamente sus inversiones en estos sectores, pero no cuentan sino con apenas siete y tres revistas (respectivamente) indexadas en Scopus. Se puede deducir que a pesar de que la 
inversión en $\mathrm{I}+\mathrm{D}$ y educación en Colombia es menor que en otros países de la región, existe un nivel de productividad alta de las instituciones y profesionales relacionados con la publicación y divulgación científica.

\section{Retos y debates actuales}

Un primer debate tiene que ver con el IBN de Publindex, corporación que además de impulsar la producción científica colombiana desde 2002, actualmente es el centro de críticas por la falta de homologación y redundancia con respecto a los índices internacionales y la pertinencia de su continuidad (Arango, 2009; Revista Semana, 2017). Igualmente, se debate sobre la conveniencia de implementar los actuales indicadores y métodos bibliométricos para la medición del impacto de la ciencia, dado que el impacto y el desarrollo científico no se pueden medir únicamente por el nivel de citas o autocitas de un autor o una revista, y de esta manera, estos indicadores no son suficientes ni para medir el impacto real de la ciencia ni para tomar decisiones de política de investigación o de presupuesto dentro de los países e instituciones de investigación y desarrollo.

Más de la cuarta parte de la producción colombiana en ciencias económicas ha trascendido la frontera nacional, aumentando su reconocimiento, visibilidad, divulgación e impacto internacional, consolidando redes científicas internacionales y procesos de calidad editorial, científica e impacto de los documentos que allí se publican. La escasa participación de la producción científica colombiana en WoS y en Scopus evidencia las dificultades que enfrentan las publicaciones colombianas para acceder en los índices promovidos por los países desarrollados.

El segundo debate se relaciona con el movimiento mundial por el acceso abierto que durante más de 15 años ha promovido la eliminación a las barreras para acceder al conocimiento científico y la información, entendidos como bienes comunes o derechos humanos (allí encontramos a DOAJ, SciELO y Redalyc), frente a las bases bibliográficas internacionales impulsadas por los países de la llamada "gran corriente de la ciencia" (donde encontramos a WoS, Scopus, Ebsco y Proquest), las cuales cobran por la suscripción y el acceso a sus contenidos (BOAI, 2012), y que además manifiestan la existencia de restricciones para el acceso y flujo de la producción científica latinoamericana en estas bases bibliográficas, afectando la visibilidad e impacto de las publicaciones colombianas en esta corriente de países emergentes (Aguado-López y Rogel-Salazar, 2006; Aguado-López et al., 2008; Tejedor-Estupiñán, 2015). Se destaca que $100 \%$ de estas revistas son de acceso abierto (Open Access), se 
publican en español (aunque algunas publican documentos en otros idiomas) y, en su mayoría, manejan soporte impreso y electrónico gestionado por la plataforma de gestión de revistas Open Journal System (OJS).

Un tercer debate tiene que ver con los recursos destinados para el sector estratégico de investigación y desarrollo (I+D), en particular para financiar el desarrollo de las revistas científicas colombianas, las cuales a pesar de los bajos recursos y limitantes han logrado posicionarse a nivel internacional (Banco Mundial, 2018; Lucio-Arias et al., 2014).

\section{Conclusiones}

Por medio de indicadores de impacto y visibilidad de la producción científica, este artículo elabora un censo de las revistas colombianas de ciencias económicas reconocidas a nivel nacional e internacional. Las revistas incluidas en este trabajo son una fuente de referencia para investigadores de todo el mundo, interesados en publicar en las revistas colombianas y latinoamericanas de ciencias económicas reconocidas a nivel internacional tanto por sus buenas prácticas científicas y editoriales como por la calidad de sus contenidos, y así, sus documentos no caigan en publicaciones de dudoso impacto, visibilidad y reconocimiento institucional. Los resultados de esta investigación son una fuente de información para editores, gestores de bases de datos y asesores del campo de la información científica y editorial.

Las revistas colombianas, en particular las relacionadas con las ciencias económicas, se han adaptado al proceso de globalización de la información en las últimas décadas, encontrando nuevos retos para el estado y las comunidades científicas. La brecha, en relación a inversión en sectores estratégicos como $\mathrm{I}+\mathrm{D}$ y educación y demás barreras que enfrentan los países en vías de desarrollo para el acceso y divulgación del conocimiento científico, deja como consecuencia que las instituciones de investigación y educación latinoamericanas estén lejos de los primeros lugares de los rankings internacionales. Esta brecha del desarrollo se puede reducir por medio del diseño y aplicación de políticas públicas adecuadas, creadas por medio de procesos de diálogo, integración y cooperación interinstitucional y regional, que posibiliten los recursos humanos y financieros necesarios para su exitosa implementación.

A pesar de las barreras descritas, más de una tercera parte de las revistas colombianas de ciencias económicas han sido clasificadas por índices internacionales de países desarrollados, y aunque hacen parte de los cuartiles de impacto medio y bajo, el mérito de llegar hasta allí ha permitido incrementar el posicionamiento y participación de instituciones de investigación 
y universidades colombianas en los rankings de producción científica y de universidades más reconocidos a nivel internacional. Se evidencia también que la mayoría de las revistas colombianas de economía se visibilizan en hemerotecas regionales como SciELO y Redalyc, y en bases de datos especializadas como RePEc, EconLit, Ebsco y Proquest.

Desde 2002, el IBN Publindex ha promovido la clasificación y evaluación de las revistas científicas colombianas y de la región. En 2016, luego de la implementación de un nuevo modelo homologado de acuerdo a estándares internacionales, eliminó de su base más de $50 \%$ de las revistas reconocidas en este índice, lo cual ha generado debates sobre su futuro, debido al impacto real del IBN para medir la producción científica colombiana y regional.

Finalmente, se destaca la diversidad de indicadores existentes para medir y analizar la productividad científica regional, nacional e institucional, así como sus limitaciones al medir el impacto de la ciencia, dado que estos indicadores abarcan los campos bibliométricos y no reflejan necesariamente el impacto real de la producción investigativa en la evolución de la ciencia, ni en el desarrollo humano y social. Los límites de los análisis bibliométricos en la medición del impacto de la ciencia presentan la cienciometría como una disciplina emergente que debe desarrollar indicadores alternativos, globales y de acceso abierto, que permitan complementar el análisis bibliométrico para sustentar presupuestos, políticas de I+D y políticas laborales más coherentes a las realidades y contextos particulares de los países y regiones, las cuales no discriminen y sí promuevan los procesos científicos generados desde las naciones emergentes.

Agradecimientos

Este documento forma parte de las actividades de investigación desarrolladas por los autores en el Grupo Finanzas y Política Económica de la Universidad Católica de Colombia (UCatólica). Los autores agradecen a la UCatólica por la colaboración para el desarrollo de esta investigación y se declaran responsables de los comentarios y contenidos de este documento.

\section{REFERENCIAS}

Abad-García, María-Francisca, Aurora González-Teruel, Javier Argento y Josep-Manuel Rodríguez-Gairín. 2015. "Características y visibilidad de las revistas españolas de ciencias de la salud en bases de datos". El profesional de la información 24 (5):537-550. https://doi.org/10.3145/epi.2015.sep.04 
Aguado-López, Eduardo y Rosario Rogel-Salazar. 2006. "Redalyc: Red de Revistas Científicas de América Latina, El Caribe, España y Portugal. Un Balance a Tres Años de Camino", en CLACSO Consejo Latinoamericano de Ciencias Sociales, editado por Dominique Babini y Jorge Fraga, 209-233. Buenos Aires: Consejo Latinoamericano de Ciencias Sociales. http://bibliotecavirtual.clacso.org.ar/ar/libros/secret/babini/Aguado Lopez Salazar.pdf

Aguado-López, Eduardo, Rosario Rogel-Salazar, Gustavo Garduño-Oropeza y María-Fernanda Zúñiga. 2008. "Redalyc: una alternativa a las asimetrías en la distribución del conocimiento científico". Ciencia, Docencia y Tecnología XIX (37): 11-30. http://www.scielo.org.ar/scielo.php?script=sci_arttext\&pi$\mathrm{d}=\mathrm{S} 1851-17162008000200002 \& \operatorname{lng}=\mathrm{es} \& \mathrm{nrm}=\mathrm{iso} \& \operatorname{lng}=\mathrm{en}$

Álvarez-Muñoz, Patricio y Mario Pérez-Montoro. 2015. "Análisis de la producción y de la visibilidad científica de Ecuador en el contexto andino (2000-2013)”. El profesional de la información 24 (5): 577-586. https://doi.org/10.3145/epi.2015.sep.07

Arango, Pablo. 2009. "La farsa de las publicaciones universitarias". El Malpensante 97: 1-5. http://www.elmalpensante.com/articulo/1031/la_farsa_de_las_publicaciones_universitarias

Archambault, Éric y Vincent Larivière. 2009. "History of the Journal Impact Factor: Contingencies and Consequences”. Scientometrics 79 (3): 635-649. https://doi. org/10.1007/s11192-007-2036-x

Babini, Dominique. 2011. “Acceso abierto a la producción científica de América Latina y El Caribe. Identificación de principales instituciones para estrategias de integración regional". Revista Iberoamericana de Ciencia, Tecnología y Sociedad CTS 6 (17): 1-24. http://www.scielo.org.ar/pdf/cts/v6n17/v6n17a03.pdf

Banco Mundial. 2018. "Gasto en investigación y desarrollo (\% del PIB)". Indicadores de Desarrollo Mundial. 2018. http://datos.bancomundial.org/indicador/GB.XPD.RSDV.GD.ZS

Bartneck, Christoph y Servaas Kokkelmans. 2011. "Detecting h-Index manipulation through self-citation analysis”. Scientometrics 87 (1): 85-98. https://doi.org/10.1007/ s11192-010-0306-5

Beltrán-Galvis, Oscar-Alfredo. 2006. "Factor de impacto”. Revista Colombiana de Gastroenterología 21 (1): 57-61. http://www.scielo.org.co/pdf/rcg/v21n1/v21n1a09.pdf

BOAI. 2012. "Ten Years on from the Budapest Open Access Initiative: Setting the Default to Open (BOAI10)". Italian Journal of Library E Information Science 3 (2): 1-15. https://doi.org/10.4403/jlis.it-8631

Charum, Jorge. 2004. "La construcción de un Sistema Nacional de Indexación. El caso de Publindex”. Convergencia 11 (35): 293-309. http://redalyc.uaemex.mx/redalyc/src/inicio/ArtPdfRed.jsp?iCve $=10503511$

Colciencias. 2013. "Indexación de revistas seriadas de Ciencia, Tecnología e Innovación colombianas". Indice Bibliográfico Nacional-Publindex. Bogotá: Colciencias. http://www.usbcali.edu.co/sites/default/files/guia-servicio-indexacion-2013.pdf

Colciencias. 2016. "Revistas indexadas - Índice Bibliográfico Nacional - Publindex 2da Actualización 2014”. Bogotá. http://scienti.colciencias.gov.co:8084/publindex/EnIbnPublindex/buscador.do

Colciencias. 2017a. "Listado de revistas clasificadas Convocatoria 768 de 2016". Bogotá. http://www.colciencias.gov.co/sites/default/files/listado-revistas-por-categoria-conv-768.pdf 
Colciencias. 2017b. "Resolución 0929. Por la cual se publican los Resultados Definitivos de la Convocatoria 768 de 2016 "para la indexación de revistas especializadas de Ciencia, Tecnología e Innovación-Publindex"”. Bogotá. http://www.colciencias.gov.co/sites/default/files/resolucion-0929-2017.pdf

Espinosa, Karem, Jairo-Humberto Restrepo y Sandra Rodríguez. 2003. "Producción académica en economía de la salud en Colombia, 1980-2002”. Lecturas de Economía 59: 7-53. http://www.redalyc.org/articulo.oa?id=155218004001

Falagas, Mattew E., Vasilios D. Kouranos, Ricardo Arencibia-Jorge y Drosos E. Karageorgopoulos. 2008. "Comparison of SCImago Journal Rank Indicator with Journal Impact Factor". The FASEB Journal 22 (8): 2623-2628. https://doi. org/10.1096/fj.08-107938

Garfield, Eugene. 1994. "The Clarivate Analytics Impact Factor". Clarivate Analytics. 1994. https://clarivate.com/essays/impact-factor/.

Garfield, Eugene. 1999. "Journal Impact Factor: A Brief Review”. Canadian Medical Association Journal - Journal de l'Association Medicale Canadienne 161 (8): 979980. https://doi.org/papers2://publication/uuid/10FB855D-C2C7-4997-BF43$7287241 \mathrm{C} 6532$

Garfield, Eugene. 2006. "The History and Meaning of the Journal Impact Factor". Journal of the American Medical Association 295 (1): 90-93. https://jamanetwork. com/journals/jama/article-abstract/202114? redirect=true

Grupo Scimago. 2006. "El índice h de Hirsch: aportaciones a un debate”. El professional de la información 15 (4): 304-306. https://doi.org/10.3145/epi.2006.jul.08

Guerrero-Bote, Vicente P. y Félix Moya-Anegón. 2012. "A Further Step Forward in Measuring Journals' Scientific Prestige: The SJR2 Indicator”. Journal of Informetrics 6 (4): 674-688. https://doi.org/10.1016/j.joi.2012.07.001

Harzing, Anne-Wil y Ron Van der Wal. 2009. "A Google Scholar h-Index for Journals: An Alternative Metric to Measure Journal Impact in Economics \& Business". Journal of the American Society for Information Science and Technology 60 (1): 41-46. https://doi.org/10.3354/esep00076

Hecht, Frederick, Barbara K. Hecht y Avery A. Sandberg. 1998. "The Journal 'Impact Factor': A Misnamed, Misleading, Misuse Measure”. Cancer Genetics and Cytogenetics 104 (2): 77-81. https://doi.org/http://dx.doi.org/10.1016/S0165-4608(97)00459-7

Hirsch, Jorge E. 2005. "An Index to Quantify an Individual's Scientific Research Output". Proceedings of the National Academy of Sciences 102 (46): 16569-16572. https://doi.org/10.1073/pnas.0507655102

Kurmis, Andrew P. 2003. "Understanding the Limitations of the Journal Impact Factor". The Journal of Bone E Joint Surgery 85-A (12): 2449-2454. https://insights.ovid.com/pubmed?pmid=14668520

Lucio-Arias, Diana, Sandra-Carolina Rivera-Torres, Jinneth Tique-Ortiz, Nelson-Fabián Villarreal, Jorge Lucio, Henry Mora, Gloria-Inés Perea et al. 2014. Indicadores de Ciencia y Tecnología Colombia 2014, editado por Jorge Lucio, Nelson Fabián Villarreal, Diana Lucio-Arias y Andrea Guevara. Bogotá: Observatorio Colombiano de Ciencia y Tecnología. http://ocyt.org.co/es-es/InformeAnualIndicadores/ArtMID/542/ArticleID/250/Indicadores-de-Ciencia-y-Tecnolog237a-Colombia-2014

Marenco-Escuderos, Ailed-Daniela. 2013. "Redes de cooperación científica entre autores e indicadores bibliométricos de una revista de psicología en Colombia (Universitas Psychologica) durante el periodo 2009-2012”. Realitas, Revista de Ciencias 
Sociales, Humanas y Artes 1 (1): 25-31. http://unireformada.edu.co/index.php/ numeros-anteriores/2013/ene-jun-vol-1-n-1/45-inicio/realitas/157-redes-de-cooperacion-cientifica-entre-autores-e-indicadores-bibliometricos-de-una-revista-de-psicologia-en-colombia

Miguel, Sandra. 2011. "Revistas y producción científica de América Latina y El Caribe: su visibilidad en SciELO, RedALyC y SCOPUS”. Revista Interamericana de Bibliotecología 34 (2): 187-199. http://www.redalyc.org/articulo.oa?id=179022554006

Montilla-Peña, Leomar-José. 2012. "Análisis bibliométrico sobre la producción científica archivística en la Red de Revistas Científicas de América Latina y El Caribe (Redalyc) durante el periodo 2001-2011”. Biblios 48: 1-11. https://doi.org/10.5195/ biblios.2012.65

Ordóñez M., Gonzalo, Alejandro Hernández B., Carolina Hernández y Carolina Mendez. 2009. "Análisis bibliométrico de la Revista de Economía Institucional en sus primeros diez años”. Revista de Economía Institucional 11 (20): 309-353. http://www.economiainstitucional.com/pdf/no20/gordonez20.pdf

Palucci-Marziale, María-Helena. 2011. "Indicadores de la producción científica iberoamericana”. Revista Latino-Americana de Enfermería 19 (4): 853-854. http:// www.scielo.br/pdf/rlae/v19n4/es_01.pdf

Revista Semana. 2017. "Para mejorar la calidad". http://www.semana.com/vida-moderna/articulo/para-mejorar-la-calidad/541550

Rodríguez, Esmeralda, Sandra Naranjo y Dory-Luz González. 2015. "Publindex: más que un proceso de indexación”. Ágora U.S.B. 15 (1): 29-41. http://www.scielo.org.co/pdf/agor/v15n1/v15n1a02.pdf

Romero-Torres, Mauricio, Luis-Alberto Acosta-Moreno y Maria-Alejandra Tejada-Gómez. 2013. "Ranking de revistas científicas en Latinoamérica mediante el índice h: estudio de caso Colombia”. Revista Española de Documentación Cientifca 36 (1): 1-13. https://doi.org/http://dx.doi.org/10.3989/redc.2013.1.876

Salvador-Oliván, José Antonio, Gonzalo Marco-Cuenca y Rosario Arquero-Avilés. 2018. "Impacto de las revistas españolas de biblioteconomía y documentación y repercusión de las autocitas en su índice h". Investigación Bibliotecológica: archivonomía, bibliotecología e información 32 (77): 13-30. http://dx.doi.org/10.22201/ iibi.24488321xe.2018.77.57852

Sánchez-Perdomo, Rubén, Marinelsy Rosario-Sierra, Darlenis Herrera-Vallejera, Yaniris Rodríguez-Sánchez y Humberto Carrillo-Calvet. 2018. "Revisión bibliométrica de las ciencias de la información en América Latina y El Caribe”. Investigación Bibliotecológica: archivonomia, bibliotecología e información (Número Especial de Bibliometría): 79-100. https://doi.org/10.22201/iibi.24488321xe.2017. nesp1.57886

SCImago Journal \& Country Rank. 2018. "SCImago Journal \& Country Rank Economics”. SCImagoLab. http://www.scimagojr.com/journalrank.php?area=2000\&country $=\mathrm{CO}$

Scopus. 2018. “CiteScore”. Journal Metrics. 2018. https://journalmetrics.scopus.com Szentes, Tamás. 2003. World Economics 2.The Political Economy of Development. Globalisation and System Transformation. Budapest: Akadémiai Kiadó.

Tejedor-Estupiñán, Joan-Miguel. 2015. "The Future of Economic Sciences Journals in Colombia”. Revista Finanzas y Política Económica 7 (2): 221-224. https://doi. org/10.14718/revfinanzpolitecon.2015.7.2.1 
Tol, Richard S. J. 2009. "The h-Index and Its Alternatives: An Application to the 100 Most Prolific Economists". Scientometrics 80 (2): 317-324. https://doi. org/10.1007/s11192-008-2079-7

Web of Science. 2017. 2017 Journal Citation Reports. Journals in the 2017 Release of JCR. London. http://images.info.science.thomsonreuters.biz/Web/ThomsonReutersScience/\%7Bda895e0c-0d4f-44f2-a6d5-6548d983a79f\%7D_M151_Crv_ JCR_Full_Marketing_List_A4_FA.pdf

Anexos

\begin{tabular}{|c|c|c|c|}
\hline Núm & Cat. & Título de la revista & Institución editora \\
\hline 1 & B & Finanzas y Politica Económica & Universidad Católica de Colombia - UCC \\
\hline 2 & B & Lecturas de Economía & Universidad de Antioquia - UdeA \\
\hline 3 & B & Apuntes del CENES & $\begin{array}{l}\text { Universidad Pedagógica y Tecnológica } \\
\text { de Colombia }\end{array}$ \\
\hline 4 & B & Cuadernos de Administración & Pontificia Universidad Javeriana \\
\hline 5 & B & Cuadernos de Desarrollo Rural & Pontificia Universidad Javeriana \\
\hline 6 & B & Estudios Gerenciales & Universidad ICESI \\
\hline 7 & B & Revista de Economía Institucional & Universidad Externado De Colombia \\
\hline 8 & B & Sociedad y Economía & Universidad del Valle - Univalle \\
\hline 9 & B & Cuadernos de Administración & Universidad del Valle - Univalle \\
\hline 10 & B & Semestre Económico & Universidad de Medellín - UDEM \\
\hline 11 & B & $\begin{array}{l}\text { Revista Escuela de Administración } \\
\text { de Negocios }\end{array}$ & Universidad EAN \\
\hline 12 & B & $\begin{array}{l}\text { Revista Facultad de Ciencias } \\
\text { Económicas }\end{array}$ & $\begin{array}{l}\text { Universidad Militar Nueva Granada - } \\
\text { Unimilitar }\end{array}$ \\
\hline 13 & C & Cuadernos de Economía & Universidad Nacional de Colombia \\
\hline 14 & C & Innovar & Universidad Nacional de Colombia \\
\hline 15 & C & Desarrollo y Sociedad & Universidad de Los Andes - Uniandes \\
\hline 16 & C & Económicas CUC & $\begin{array}{l}\text { Corporación Universidad de La Costa } \\
\text { CUC }\end{array}$ \\
\hline 17 & C & Ensayos de Economía & Universidad Nacional de Colombia \\
\hline 18 & C & Equidad \& Desarrollo & Universidad de La Salle - Unisalle \\
\hline 19 & C & Clio América & Universidad del Magdalena \\
\hline 20 & C & Panorama Económico & Universidad de Cartagena \\
\hline
\end{tabular}

Anexo 1. Revistas de ciencias económicas clasificadas en el IBN de Publindex-Colciencias, 2017 


\begin{tabular}{|c|c|c|c|c|c|c|c|c|c|c|}
\hline \multirow{4}{*}{ 옹 } & ¿̊ & $\dot{0}$ & 宁 & $\underset{\omega}{\infty}$ & $\widehat{r o}$ & $\stackrel{10}{\mathrm{~N}}$ & $\stackrel{+}{\sim}$ & $\stackrel{\infty}{\underset{\varepsilon}{+}}$ & 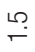 & ○ \\
\hline & 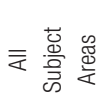 & $\underset{\square}{\check{\Xi}}$ & $\underset{\substack{\mathcal{L} \\
\sim}}{\mathbb{P}}$ & $\stackrel{\circ}{\circ}$ & 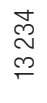 & $\underset{\infty}{\mathfrak{⿰}}$ & $\underset{\infty}{\mathbb{N}}$ & $\begin{array}{l}\underset{D}{\sim} \\
\stackrel{\sim}{\square}\end{array}$ & क्లे & $\frac{\Gamma}{\frac{\pi}{J}}$ \\
\hline & $\partial^{\circ}$ & $\stackrel{\nabla}{\circ}$ & $\hat{m}$ & $\begin{array}{l}0 \\
\oplus \\
0\end{array}$ & $\overline{6}$ & 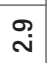 & ஜ̊ & $\stackrel{\sim}{\sim}$ & $\digamma$ & ঃ \\
\hline & 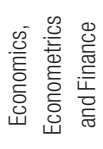 & $\nabla$ & $\hat{m}$ & $\mathscr{8}$ & $\frac{\nabla}{6}$ & న & $\sigma$ & $\widehat{\widetilde{N}}$ & $F$ & $\stackrel{0}{0}$ \\
\hline \multirow{4}{*}{ 오 } & $\circ$ & $\begin{array}{l}0 \\
0 \\
0\end{array}$ & $\begin{array}{l}\infty \\
\infty \\
\infty\end{array}$ & 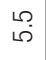 & $\begin{array}{l}\text { ఠ) } \\
\stackrel{\infty}{\sigma}\end{array}$ & 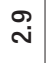 & $\stackrel{\circ}{\sim}$ & ন্ঠ & $\stackrel{\sim}{\sim}$ & ○ \\
\hline & 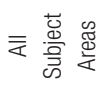 & g) & 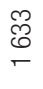 & $\begin{array}{l}\stackrel{0}{\triangle} \\
\stackrel{\Xi}{-}\end{array}$ & $\begin{array}{l}\text { ? } \\
\text { ᄋ } \\
0\end{array}$ & 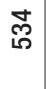 & $\underset{m}{\stackrel{\infty}{m}}$ & 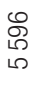 & 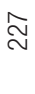 & $\begin{array}{l}\overline{6} \\
6 \\
\infty \\
\varnothing\end{array}$ \\
\hline & $\overbrace{}^{\circ}$ & $\ddot{\circ}$ & $\widehat{\sim}$ & $\stackrel{\leftrightarrow}{\circ}$ & $\frac{0}{6}$ & $\stackrel{m}{m}$ & $\stackrel{0}{0}$ & 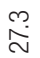 & $\stackrel{\sim}{\leftarrow}$ & 음 \\
\hline & 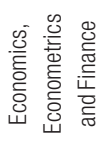 & $\sim$ & $\stackrel{\infty}{\leftarrow}$ & $\hat{\sim}$ & $\stackrel{\sim}{\longleftarrow}$ & $\approx$ & $\sim$ & $\underset{\sim}{\not}$ & $\infty$ & $\frac{12}{6}$ \\
\hline \multirow{4}{*}{$\stackrel{\circ}{\circ}$} & 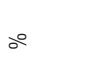 & مْ & $\underset{\infty}{\sim}$ & 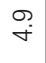 & $\stackrel{\widehat{o}}{\stackrel{\infty}{\sigma}}$ & $\stackrel{\varphi}{\leftarrow}$ & $\stackrel{\circ}{\circ}$ & ๗ें & $\stackrel{?}{\rightleftharpoons}$ & 음 \\
\hline & 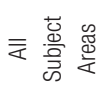 & 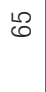 & $\stackrel{\circ}{\stackrel{\circ}{\leftarrow}}$ & \begin{tabular}{l}
10 \\
$\infty$ \\
\hdashline
\end{tabular} & $\begin{array}{l}0 \\
\infty \\
0 \\
0\end{array}$ & ల్లి & $\underset{\simeq}{\cong}$ & $\underset{\substack{\infty \\
\sim}}{\mathscr{\sigma}}$ & $\widehat{\underline{\theta}}$ & $\begin{array}{l}\stackrel{\infty}{O} \\
\stackrel{\square}{\square}\end{array}$ \\
\hline & $\therefore$ & $\stackrel{\circ}{\circ}$ & $\stackrel{+}{\leftarrow}$ & 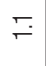 & 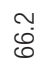 & $\stackrel{0}{0}$ & $\stackrel{\circ}{\circ}$ & ¿े. & m. & 음 \\
\hline & 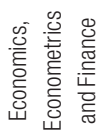 & 0 & 10 & $\nabla$ & 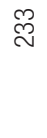 & $\sim$ & 0 & 음 & - & స్ల్ల్ \\
\hline & 듬 & 畩 & 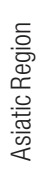 & 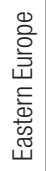 & 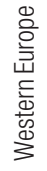 & 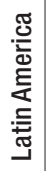 & 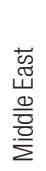 & 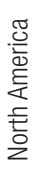 & 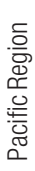 & \multirow[t]{2}{*}{ 覀 } \\
\hline & & - & $\sim$ & $m$ & $\forall$ & 10 & 0 & $\wedge$ & $\infty$ & \\
\hline
\end{tabular}




\begin{tabular}{|c|c|c|c|c|c|}
\hline Rank & Title & SJR & $\begin{array}{l}\text { SJR Best } \\
\text { Quartile }\end{array}$ & $\boldsymbol{h}$-index & Country \\
\hline 1 & Revista Brasileira de Economía & 0.311 & Q2 & 9 & Brasil \\
\hline 2 & Brazilian Journal of Political Economy & 0.282 & Q2 & 13 & Brasil \\
\hline 3 & Revista de Economia e Sociologia Rural & 0.265 & Q3 & 11 & Brasil \\
\hline 4 & Journal of Applied Economics & 0.235 & Q2 & 16 & Argentina \\
\hline 5 & Economía Aplicada & 0.208 & Q3 & 7 & Brasil \\
\hline 6 & Estudios Económicos & 0.204 & Q3 & 8 & Brasil \\
\hline 7 & Trimestre Económico & 0.173 & Q4 & 9 & México \\
\hline 8 & Nova Economía & 0.156 & Q3 & 5 & Brasil \\
\hline 9 & Cuadernos de Desarrollo Rural & 0.154 & Q4 & 6 & Colombia \\
\hline 10 & Social and Economic Studies & 0.148 & Q4 & 5 & Jamaica \\
\hline 11 & Revista Contabilidade \& Finanças & 0.145 & Q4 & 1 & Brasil \\
\hline 12 & Revista de Economía Institucional & 0.141 & Q4 & 5 & Colombia \\
\hline 13 & América Latina en la Historia Económica & 0.134 & Q4 & 3 & México \\
\hline 14 & Investigación Económica & 0.132 & Q4 & 6 & México \\
\hline 15 & Latin American Journal of Economics & 0.127 & Q4 & 10 & Chile \\
\hline 16 & Problemas del Desarrollo & 0.123 & Q4 & 4 & México \\
\hline 17 & CEPAL Review & 0.122 & Q4 & 10 & Chile \\
\hline 18 & Estudios de Economía & 0.121 & Q4 & 10 & Chile \\
\hline 19 & Revista de Economía Contemporánea & 0.121 & Q4 & 5 & Brasil \\
\hline 20 & Revista de Economía del Rosario & 0.118 & Q4 & 3 & Colombia \\
\hline 21 & $\begin{array}{l}\text { Innovar: Revista de Ciencias Administrati- } \\
\text { vas y Sociales }\end{array}$ & 0.118 & Q4 & 7 & Colombia \\
\hline 22 & Cuadernos de Administración & 0.118 & Q4 & 6 & Colombia \\
\hline 23 & Economía Chilena & 0.117 & Q4 & 4 & Chile \\
\hline 24 & Cuadernos de Economía & 0.115 & Q4 & 4 & Colombia \\
\hline 25 & Revista de Análisis Económico & 0.112 & Q4 & 4 & Chile \\
\hline 26 & Agroalimentaria & 0.111 & Q4 & 5 & Venezuela \\
\hline 27 & Desarrollo y Sociedad & 0.109 & Q4 & 3 & Colombia \\
\hline 28 & $\begin{array}{l}\text { Desarrollo Económico: Revista de Cien- } \\
\text { cias Sociales }\end{array}$ & 0.104 & Q4 & 15 & Argentina \\
\hline 29 & Revista Finanzas y Política Económica & - & - & 0 & Colombia \\
\hline
\end{tabular}

Anexo 3. Revistas latinoamericanas de ciencias económicas en SJR 2018 


\begin{tabular}{|c|c|c|c|c|c|c|c|c|}
\hline 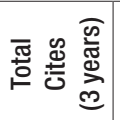 & $\stackrel{m}{m}$ & $\infty$ & $\wedge$ & 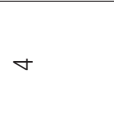 & ल & 으 & $\sim$ & ' \\
\hline 要 & $\widetilde{\text { ల్ల }}$ & $\stackrel{\infty}{\leftarrow}$ & 怘 & & $\begin{array}{l}\underset{\sim}{0} \\
\stackrel{\infty}{\sim} \\
\sim\end{array}$ & \begin{tabular}{l}
$\infty$ \\
\hdashline \\
$\infty$ \\
$-\infty$
\end{tabular} & $\frac{m}{\infty}$ & 造 \\
\hline 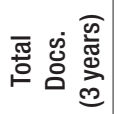 & ש్ర & 음 & L? & $\bar{m}$ & $\ddot{\mathscr{\theta}}$ & $\stackrel{2}{\circ}$ & $\hat{m}$ & $\stackrel{\infty}{\sim}$ \\
\hline 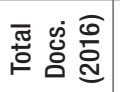 & 0 & 寸 & $\infty$ & $\nabla$ & $\mathscr{q}$ & $\stackrel{\infty}{\infty}$ & $\mathscr{\circ}$ & 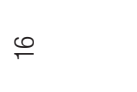 \\
\hline 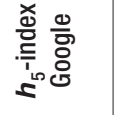 & 으 & $\mp$ & 으 & $\frac{\bar{D}}{\infty}$ & $\simeq$ & $\infty$ & $\wedge$ & ' \\
\hline 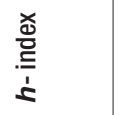 & ما & $\nabla$ & م & $m$ & 0 & $m$ & $m$ & ' \\
\hline 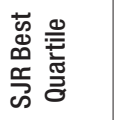 & $\mathscr{8}$ & $\mathscr{8}$ & $\mathscr{8}$ & ठ & 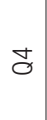 & ठ & ठ & ' \\
\hline 站 & 占 & $\begin{array}{l}\infty \\
\circ \\
0\end{array}$ & $\frac{1}{\circ}$ & $\frac{m}{\circ}$ & $\begin{array}{l}\stackrel{̊}{0} \\
\stackrel{0}{0}\end{array}$ & E & 농 & 0 \\
\hline 号 & 苫 & $\underset{\frac{\sigma}{5}}{\stackrel{5}{0}}$ & $\stackrel{\infty}{\frac{\infty}{\circ}}$ & $\underset{\leftarrow}{\risingdotseq}$ & $\underset{\leftarrow}{\stackrel{\infty}{\circ}}$ & $\stackrel{10}{\frac{10}{0}}$ & $\frac{8}{\circ}$ & 읃 \\
\hline $\begin{array}{l}\text { ల్ } \\
\underline{\underline{p}}\end{array}$ & 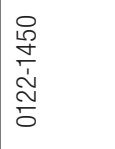 & 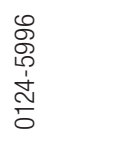 & 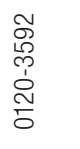 & 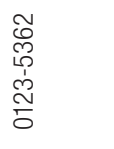 & $\begin{array}{l}\bar{L} \\
\stackrel{5}{0} \\
\frac{1}{\sim} \\
\tilde{0}\end{array}$ & 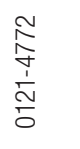 & 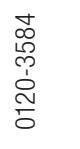 & 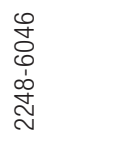 \\
\hline$\stackrel{\infty}{\stackrel{D}{E}}$ & 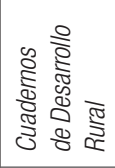 & 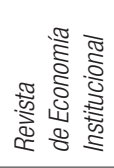 & 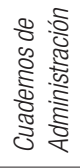 & 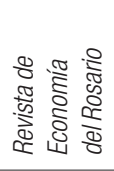 & $\begin{array}{l}\grave{\bar{s}} \\
\vdots \\
\vdots\end{array}$ & 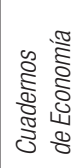 & 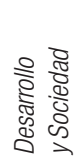 & 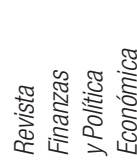 \\
\hline 䮈 & - & $\sim$ & $m$ & $\nabla$ & ما & 0 & $\wedge$ & $\infty$ \\
\hline
\end{tabular}




\begin{tabular}{|c|c|c|c|c|c|c|c|c|c|c|c|c|c|c|}
\hline 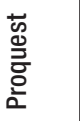 & is & 2 & 20 & 2 & is & is & 2 & ¿ & is & i & 울 & ఉ & 2 & 을 \\
\hline $\begin{array}{l}\text { 앴 } \\
\text { யि }\end{array}$ & is & in & is & ఉ & is & is & ఉ & ఉ & is & is & is & z & 2 & is \\
\hline 惡 & is & $\overline{i s}$ & 2 & is & is & $\bar{\omega}$ & is & ¿s & is & 2 & 을 & 2 & 2 & 을 \\
\hline 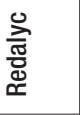 & is & is & is & ఉळ & ఉ & is & ఉ & ఉ & is & 을 & 을 & z & 2 & 2 \\
\hline 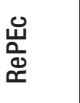 & ఉ & 일 & 2 & ఉ & ఉ & is & ఉ & ๘ & 的 & 2 & 2 & ఉ & 2 & 일 \\
\hline 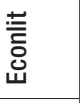 & is & 2 & 2 & is & is & is & is & ๘ & is & 2 & $\bar{c}$ & ๘ & is & is \\
\hline 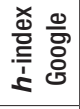 & $\stackrel{\infty}{\leftarrow}$ & $\mp$ & $\sigma$ & $\infty$ & $\wedge$ & $\sim$ & $\wedge$ & 0 & ما & م & 10 & م & $\frac{\partial}{\omega}$ & $\frac{\partial}{\omega}$ \\
\hline $\begin{array}{l}z \underline{\mathcal{W}} \\
\text { }\end{array}$ & 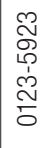 & 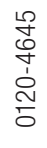 & 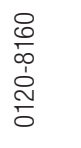 & $\begin{array}{l}12 \\
0 \\
0 \\
0 \\
1 \\
\\
\tilde{0} \\
0\end{array}$ & 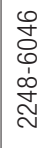 & 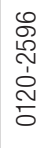 & $\begin{array}{l}\hat{L} \\
\tilde{N} \\
0 \\
1 \\
\llcorner \\
0 \\
0\end{array}$ & 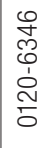 & 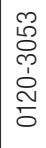 & 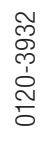 & 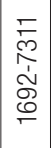 & 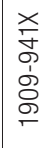 & $\underset{\frac{x}{N}}{\frac{1}{\sim}}$ & 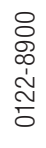 \\
\hline $\begin{array}{l}\text { 히 } \\
\text { हे }\end{array}$ & 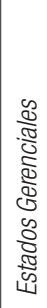 & 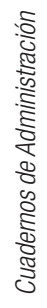 & 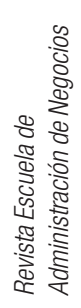 & 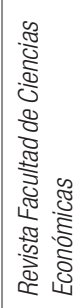 & 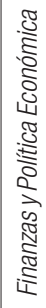 & 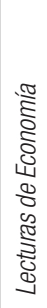 & 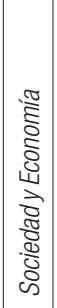 & 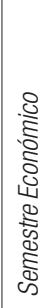 & 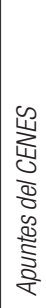 & 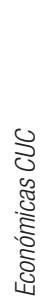 & 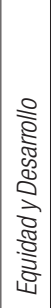 & 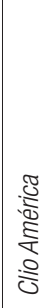 & 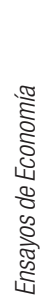 & 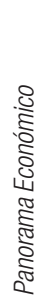 \\
\hline & - & $\sim$ & $m$ & $\nabla$ & م & 0 & 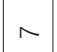 & $\infty$ & $\infty$ & 으 & $F$ & $\simeq$ & $\stackrel{m}{\longrightarrow}$ & $\underset{\ulcorner}{ }$ \\
\hline
\end{tabular}


Para citar este texto:

Tejedor-Estupiñán, Joan-Miguel y Ricardo-Alonso Tejedor-Estupiñán. 2019. "Visibilidad e impacto científico: el caso de las revistas colombianas de economía”. Investigación Bibliotecológica: archivonomía, bibliotecología e información 33 (78): 121-141.

http://dx.doi.org/10.22201/iibi.24488321xe.2019.78.57915 